Virginia Commonwealth University

VCU Scholars Compass

2016

\title{
Role of the Calcium Plateau in the Neuronal Injury and Behavioral Morbidities Following Organophosphate Intoxication
}

Laxmikant S. Deshpande

Virginia Commonwealth University, laxmikant.deshpande@vcuhealth.org

Robert E. Blair

Virginia Commonwealth University, robert.blair@vcuhealth.org

Kristin F. Phillips

Virginia Commonwealth University, kristin.phillips@vcuhealth.org

Robert J. DeLorenzo

Virginia Commonwealth University, robert.delorenzo@vcuhealth.org

Follow this and additional works at: http://scholarscompass.vcu.edu/neurology_pubs

Part of the Molecular and Cellular Neuroscience Commons, Neurology Commons, and the Toxicology Commons

(C) 2016 New York Academy of Sciences.

\section{Downloaded from}

http://scholarscompass.vcu.edu/neurology_pubs/16

This Article is brought to you for free and open access by the Dept. of Neurology at VCU Scholars Compass. It has been accepted for inclusion in Neurology Publications by an authorized administrator of VCU Scholars Compass. For more information, please contact libcompass@vcu.edu. 
Short title: Calcium dysregulation following organophosphate toxicity

\title{
Role of the Calcium Plateau in the Neuronal Injury and Behavioral Morbidities Following Organophosphate Intoxication
}

\author{
Laxmikant S. Deshpande a, b, Robert E. Blair, Kristin F. Phillips, and Robert J. DeLorenzo \\ a, b \\ Department of Neurology ${ }^{\mathrm{a}}$, Pharmacology and Toxicology ${ }^{\mathrm{b}}$, \\ Virginia Commonwealth University, Richmond, VA 23298, USA \\ To whom correspondence should be addressed: \\ Robert J. DeLorenzo, M.D., Ph.D., M.P.H. \\ Virginia Commonwealth University \\ School of Medicine \\ PO Box 980599 \\ Richmond, VA 23298 \\ Phone: 804-828-3392 \\ Fax: 804-828-6432 \\ E-mail: Robert.DeLorenzo@,vcuhealth.org
}

Keywords: Paraoxon; Status Epilepticus; Cell death; Calcium; Dantrolene; Carisbamate 


\begin{abstract}
:
Organophosphate (OP) chemicals include nerve agents and pesticides, and there is a growing concern of OP based chemical attacks against civilians. Current antidotes are essential in limiting immediate mortality associated with OP exposure. However, further research is needed to identify molecular mechanisms underlying long-term neurological deficits following survival of OP toxicity in order to develop effective therapeutics. We have developed rat survival models of OP induced status epilepticus (SE) that mimic chronic mortality and morbidity following OP intoxication. We have observed significant elevations in hippocampal calcium levels after OP SE that persisted for weeks following initial survival. Drugs inhibiting intracellular calcium-induced calcium release such as dantrolene, levetiracetam, and carisbamate lowered OP-SE mediated protracted calcium elevations. Given the critical role of calcium signaling in modulating behavior and cell-death mechanisms, drugs targeted at preventing the development of the calcium plateau could enhance neuroprotection, help reduce morbidity and improve outcome following survival of OP SE.
\end{abstract}




\section{Introduction}

\section{The Increasing Risk for Organophosphate Exposure}

Organophosphate (OP) chemicals include nerve agents such as Sarin and pesticides such as Parathion. These compounds are considered extremely lethal. The civilian population has been exposed to nerve agents under acts of war and terrorism. Recent examples include the reported 2015 Sarin gas attack in Ghouta, Syria ${ }^{1}$, the Tokyo sub-way Sarin attack by the "Aum Shinrikyo" cult in $1995^{2}$, and the 1988 "Halabja chemical attack" against Kurdish people in Iraq ${ }^{3}$. OP based pesticides have also been used against civilians during the Rhodesian $\mathrm{War}^{4}$ and the accidental poisoning in Indian children following consumption of pesticide contaminated lunches

5 . In addition, civilians are exposed to OP's intentionally by suicide attempts or occupationally or due to industrial accidents. In fact, pesticide ingestion is one of the most common method for committing suicide in developing nations ${ }^{6,78}$. The military population has also been exposed to OP chemicals. Approximately $30 \%$ of returning soldiers from the Persian Gulf War suffer from a cluster of symptoms commonly known as Gulf War Syndrome. Prolonged exposure to OP based pesticides or exposure to Sarin gas, following demolition of chemical weapon stockpiles are amongst the possible causes thought to be responsible for this syndrome ${ }^{9-11}$. The ease of availability of pesticides make them attractive target to be weaponized and cause mass civilian causalities. Thus, there is a growing threat of OP toxicity in the current geopolitical environment. Research in this field has provided therapeutic antidotes that are critical in limiting immediate mortality associated with lethal OP intoxication ${ }^{12}$. However, further research is needed to identify molecular mechanisms underlying chronic mortality and morbidity in order to develop effective counteract therapeutics following OP exposure ${ }^{13}$. 


\section{Organophosphate Poisoning: Mechanisms, Treatments, and Challenges}

Paraoxon (POX) is an active metabolite of parathion and is used in laboratory research to reliably model OP pesticide toxicity ${ }^{14}$. Similarly, diisopropyl fluorophosphates (DFP) is used in civilian research as a nerve agent surrogate to model sarin exposure given the ease of handling associated with DFP ${ }^{15-18}$. POX, DFP and other OP chemicals are potent inhibitors of the enzyme acetylcholinesterase $(\mathrm{AChE})^{19}$. Inhibition of $\mathrm{AChE}$ prevents breakdown of the neurotransmitter acetylcholine $(\mathrm{ACh})$ and rapidly builds up ACh level at the synapses. Overstimulation of ACh receptors leads to the classical "cholinergic crisis" characterized by salivation, lacrimation, urination, and defecation. This is followed by respiratory depression and bradycardia. Nicotinic receptor stimulation causes muscle fasciculation. This is followed by tonic-clonic seizures and status epilepticus (SE), or prolonged seizure activity that continues unabated and results in the death if left untreated ${ }^{20}$. SE activity is thought to involve recruitment of N-methyl-D-aspartate (NMDA) receptors following release of the excitatory neurotransmitter glutamate downstream of the ACh overstimulation ${ }^{21-24}$. Current treatment strategies use atropine to control the cholinergic crisis, pralidoxime, to reactivate $\mathrm{AChE}$, and a benzodiazepine such as diazepam or midazolam to control seizures ${ }^{25} 26$. While the current antidotes are critical in limiting immediate mortality associated with OP exposure, OP/ SE survivors are vulnerable to delayed mortality in the critical 2-week period post initial survival and the development of chronic neurological morbidities such as recurrent seizures, depression and cognitive deficits ${ }^{14,22,27-35}$. Thus, it is essential to develop valid animal models that mimic OP mortality and morbidity and to identify molecular mechanisms underlying long-term neurological deficits from survival of OP toxicity in order to develop effective counteract therapeutics. 


\section{Rat survival models of OP-SE}

Many OP studies in literature have focused on effects of low-dose, chronic OP exposure or effects of OP's following in-utero exposure ${ }^{36-39}$. There are also studies reporting models of acute parathion ${ }^{40,41}$ and POX exposures ${ }^{42-44}$. However, these models did not focus on evaluating long term survival after lethal POX SE exposures. Development of OP SE models is also complicated by their variable pharmacokinetic and pharmacodynamics response, such as the challenges associated with parathion kinetics and differential metabolism ${ }^{45-47}$. We wanted to further develop a reliable rat survival model for lethal OP exposure with SE that would replicate both the acute mortality and chronic morbidity associated with these agents. Such animal models could be very useful to study molecular mechanisms of OP toxicities and screen medical countermeasures to improve survival following OP exposures.

To this end we have developed two SE survival models of OP toxicity using lethal doses of POX ${ }^{14}$ and DFP ${ }^{16}$. The behavioral manifestations, and EEG profile for these OP SE models mimicked the signs and symptoms of acute OP intoxication. In this model, rats were exposed to a lethal dose (approximately $2 \times L_{50}$ ) of OP chemical (POX or DFP) and were treated with FDA approved drugs to limit immediate mortality ${ }^{26}$. Here we will discuss the POX model of OP-SE. One week prior to SE experiments, rats were stereotaxically implanted with skull surface electrodes to record EEG. Briefly, one minute following POX injection $(2 \mathrm{mg} / \mathrm{kg}$, s.c.) animals received human-dose equivalents of 2-PAM ( $25 \mathrm{mg} / \mathrm{kg}$, i.m. $)$ and atropine $(0.5 \mathrm{mg} / \mathrm{kg}$, i.m. $)$. Within 5-7 minutes following POX administration, rats displayed overt cholinergic symptoms and rapidly developed convulsions and SE-like activity. Onset of SE was determined by the presence of continuous class 4-5 level seizures using a modified Racine scale ${ }^{48}$. One hour following onset of POX SE, animals were injected with midazolam ( $2 \mathrm{mg} / \mathrm{kg}$, i.m.) to terminate 
seizures. Surviving animals were then injected with saline (3cc/animal, i.p.) and fed lactose milk as part of supportive care and returned to their home cages. Surviving rats were housed individually in temperature and light controlled vivarium. All the rats were visually monitored once a week till their use in $\mathrm{Ca}^{2+}$ imaging or behavioral experiments. Chronic mortality (72-h and beyond) in these models of severe OP intoxication was $18-20 \%{ }^{14,16}$. These POX and DFP SE survival models manifested the same degree of delayed mortality and morbidity (see below) observed in the human OP exposure condition ${ }^{49-53}$.

\section{Development of " $\mathrm{Ca}^{2+}$ Plateau” following survival from OP-SE}

One of the important long-term molecular changes that occurs following the survival of SE induced by OPs or chemoconvulsants like pilocarpine is the development of sustained elevations in neuronal calcium levels $\left(\left[\mathrm{Ca}^{2+}\right]_{\mathrm{i}}\right)$ known as the " $\mathrm{Ca}^{2+}$ plateau" ${ }^{24,16,54-59}$. We have developed methodologies to acutely isolate hippocampal CA1 region neurons from brain slices using enzymatic and mechanical trituration. Neurons obtained using these methods show minimal signs of necrosis, exhibit normal electrophysiological membrane properties, and allows us to study $\mathrm{Ca}^{2+}$ dynamics in the absence of confounding factors such as glial response. Estimation of neuronal $\mathrm{Ca}^{2+}$ levels have revealed the development of a $\mathrm{Ca}^{2+}$ plateau wherein hippocampal neurons exhibit significantly elevated $\mathrm{Ca}^{2+}$ levels for weeks after the termination of POX SE ${ }^{14}$ (Fig. 1A). We have previously shown that while the induction of $\mathrm{Ca}^{2+}$ plateau was NMDA receptor dependent during $\mathrm{SE}^{16,54}$, the maintenance of the $\mathrm{Ca}^{2+}$ plateau for several weeks post-SE was independent of NMDA receptor activation and was mediated by persistent $\mathrm{Ca}^{2+}$ release from the endoplasmic reticulum through the mechanisms of $\mathrm{Ca}^{2+}$ induced $\mathrm{Ca}^{2+}$ release $^{56,57}$. Indeed, pre-treatment with the NMDA antagonist MK-801 prevented the OP SE induced elevations in hippocampal $\mathrm{Ca}^{2+}$ levels. However, application of MK-801 was not 
effective in lowering the elevated $\mathrm{Ca}^{2+}$ in hippocampal neurons isolated from rats 1-h following $\underline{\mathrm{SE}}{ }^{16}$ (Fig. 1B). On the other hand, treatments with dantrolene or levetiracetam or carisbamate, inhibitors of the $\mathrm{Ca}^{2+}$ induced $\mathrm{Ca}^{2+}$ release mechanisms were able to lower the elevated $\mathrm{Ca}^{2+}$ levels and abolish the $\mathrm{Ca}^{2+}$ plateau post $\mathrm{SE}^{14,57,60}$ (Fig. 1C). It is important to note that while NMDA-R mediated indiscriminant $\mathrm{Ca}^{2+}$ influx turns off after SE is terminated, there remains a $\underline{\text { sustained } \mathrm{Ca}^{2+} \text { release from ER continues due to a long lasting activation of molecular }}$ components involved in the CICR mechanisms. This is an important aspect of the long lasting activation of the CICR system. Since $\mathrm{Ca}^{2+}$ ions act as major second messengers in multiple signaling cascades, the OP SE induced prolonged elevations in $\left[\mathrm{Ca}^{2+}\right]_{i}$ can trigger neurodegenerative pathways and mediate pathological synaptic plasticity. These alterations in $\mathrm{Ca}^{2+}$ dynamics following OP toxicity could therefore underlie the associated neuronal injury and together they be responsible for the chronic neurological morbidities following OP SE ${ }^{14}$ survival (Fig. 4).

\section{Neuronal Injury following OP-SE}

Neuronal loss in several brain regions has been observed following $\mathrm{SE}^{54,55}$, $\mathrm{OP} \mathrm{SE}^{14,15}$ and other chemical threat agents ${ }^{61}$. We have observed widespread neuronal loss induced by POX SE.as assessed using the Fluoro Jade (FJC) labeling technique ${ }^{14,62}$. FJC-positive staining neurons were observed within the hippocampus, parietal cortex, and in both amygdala and thalamic nuclear regions of POX SE rats (Fig. 2). Damages to these critical brain areas have been implicated in memory impairment, depression, anxiety, epilepsy and other neurological morbidities $^{27,63,64}$. 


\section{Chronic morbidity following survival from OP-SE}

We have also analyzed OP SE survivors in these animal models for the development of neurological morbidities (Fig. 3). We have observed symptoms of chronic depression and memory impairments in these OP exposed rats ${ }^{62,65}$. OP SE survivors displayed increased immobility in the Forced Swim Test indicative of despair, reduced sucrose consumption in the Sucrose Preference Test indicative of anhedonia, and spend less time in the open arm of elevated plus maze indicative of high anxiety ${ }^{62,65}$. Together, despair, anhedonia, and anxiety constituted symptoms of depression. In addition, these rats performed poorly in the Novel Object Recognition task indicative of memory impairment ${ }^{62,65}$. Survival from OP SE was also associated with significant neuronal damage throughout the limbic system, particularly the hippocampus ${ }^{14,16}$. These models provide a reproducible method of mimicking the human survival of OP toxicity. In addition to lethal OP intoxication, chronic low-dose OP exposures have also been implicated in long-term neurological morbidities. For example, agricultural pesticide applicators, and Persian Gulf War veterans suspected of chronic OP exposure exhibit chronic neurological morbidities such as depression and cognitive impairments ${ }^{33,66,67}$.

\section{Conclusion}

$\mathrm{Ca}^{2+}$ ions are second messenger molecules in various signaling cascades that modulate behavior, memory, and cell death $\mathrm{h}^{55,56,68-72}$. The development of $\mathrm{Ca}^{2+}$ plateau is therefore a critical substrate for inducing neuronal damage and triggering many of the long term plasticity changes following OP-SE induced by brain injury ${ }^{14,16,55,56}$. Given the role of $\mathrm{Ca}^{2+}$ induced $\mathrm{Ca}^{2+}$ release mechanisms in the maintenance of $\mathrm{Ca}^{2+}$ plateau, drugs targeting the molecular components of this signaling mechanisms could prove to be effective agents in extending neuroprotection following survival from OP-SE. We have demonstrated neuroprotective and 
antiepileptogenic effects of dantrolene $e^{57}$ and carisbamate ${ }^{73}$ in an in vitro model of SE induced acquired epilepsy. The ability of dantrolene, levetiracetam, and carisbamate to reduce or abolish the $\mathrm{Ca}^{2+}$ plateau could make them attractive neuroprotective adjuvant treatments following OPSE. These agents could also prove beneficial in reducing the chronic neurological morbidities observed in OP SE survivors. We are actively exploring these possibilities in our laboratories (Fig. 4).

Despite advances in developing more effective agents for controlling the cholinergic crisis associated with OP SE, there is a pressing need to develop counteract treatments that prevent or reduce the high mortality and the chronic morbidity associated with OP SE. This is an important area of research that has direct translational implications for clinical treatment ${ }^{74,75}$. Development of animal models of OP SE are critical to identifying molecular mechanisms underlying symptoms of OP toxicity. This knowledge can provide molecular targets that can be used to develop effective therapies for the treatment of OP SE (Fig. 4). This research indicated that agents that inhibit $\mathrm{Ca}^{2+}$ induced $\mathrm{Ca}^{2+}$ release and can reduce or prevent the $\mathrm{Ca}^{2+}$ plateau may be an innovative area for development of medical countermeasures that can lower mortality and morbidity following SE and OP SE. 


\section{Acknowledgements}

This work was supported by the CounterACT Program, National Institutes of Health Office of the Director (NIH OD), and the National Institute of Neurological Disorders and Stroke Grant No. (U01NS058213-10) to RJD. Its contents are solely the responsibility of the authors and do not necessarily represent the official views of the federal government. This work was also supported by the Office of the Assistant Secretary of Defense for Health Affairs through the Gulf War Illness Research Program under Award No. (W81XWH-14-1-0478) to LSD. Opinions, interpretations, conclusions, and recommendations are those of the author and are not necessarily endorsed by the Department of Defense. Portions of this work were presented at the 9th Annual NIH Countermeasures against Chemical Threats (CounterACT) Network Research Symposium. 


\section{References}

1. Sellstrom, A., S. Cairns \& M. Barbeschi. Report of the United Nations Mission to Investigate Allegations of the Use of Chemical Weapons in the Syrian Arab Republic on the alleged use of chemical weapons in the Ghouta area of Damascus on 21 August 2013. https://disarmament-library.un.org/UNODA/Library.nsf/ 780cfafd472b047785257b1000501037/e4d4477c9b67de9085257bf800694bd2/\$FILE/A\%2067\% 20997-S\%202013\%20553.pdf

2. Hood, E. 2001. The Tokyo attacks in retrospect: sarin leads to memory loss. Environ Health Perspect. 109: A542.

3. Dingeman, A. \& R. Jupa. 1987. Chemical warfare in the Iran-Iraq conflict. Strategy and Tactics Magazine. 113: 51-52.

4. Moorcraft, P. \& P. McLaughlin. 2008. The Rhodesian War: A Military History. Yorkshire: Pen \& Sword.

5. Than, K. 2013. Organophosphates: A Common But Deadly Pesticide. National Geographic. http://news.nationalgeographic.com/news/2013/07/130718-organophosphatespesticides-indian-food-poisoning/

6. Ajdacic-Gross, V., M.G. Weiss, M. Ring, et al. 2008. Methods of suicide: international suicide patterns derived from the WHO mortality database. Bulletin of the World Health Organization. 86: 726-732.

7. Konradsen, F. 2007. Acute pesticide poisoning--a global public health problem. Danish medical bulletin. 54: 58-59.

8. Blakey, D.H., M. Lafontaine, J. Lavigne, et al. 2013. A screening tool to prioritize public health risk associated with accidental or deliberate release of chemicals into the atmosphere. BMC public health. 13: 253.

9. Haley, R.W. \& J.J. Tuite. 2013. Epidemiologic evidence of health effects from longdistance transit of chemical weapons fallout from bombing early in the 1991 Persian Gulf War. Neuroepidemiology. 40: 178-189.

10. Special Assistant for Gulf War Illnesses. 2001. "Environmental Exposure Report Pesticides". http://www.gulflink.osd.mil.

11. Institute of Medicine: Board on the Health of Select Populations. 2013. Gulf War and Health: Treatment of Chronic Multisymptom Illness. http:/www.iom.edu/Reports/2013/GulfWar-and-HealthTreatment-for-Chronic-Multisymptom-Illness.aspx

12. Broomfield, C.A. \& S.D. Kirby. 2001. Progress on the road to new nerve agent treatments. J Appl Toxicol. 21 Suppl 1: S43-46.

13. Jett, D.A. 2011. Neurotoxic pesticides and neurologic effects. Neurologic clinics. 29: 667-677.

14. Deshpande, L.S., D.S. Carter, K.F. Phillips, et al. 2014. Development of status epilepticus, sustained calcium elevations and neuronal injury in a rat survival model of lethal paraoxon intoxication. Neurotoxicology. 44C: 17-26.

15. Li, Y., P.J. Lein, C. Liu, et al. 2011. Spatiotemporal pattern of neuronal injury induced by DFP in rats: a model for delayed neuronal cell death following acute OP intoxication. Toxicol Appl Pharmacol. 253: 261-269.

16. Deshpande, L.S., D.S. Carter, R.E. Blair, et al. 2010. Development of a prolonged calcium plateau in hippocampal neurons in rats surviving status epilepticus induced by the organophosphate diisopropylfluorophosphate. Toxicol Sci. 116: 623-631. 
17. Zaja-Milatovic, S., R.C. Gupta, M. Aschner, et al. 2009. Protection of DFP-induced oxidative damage and neurodegeneration by antioxidants and NMDA receptor antagonist. Toxicol Appl Pharmacol. 240: 124-131.

18. Pibiri, F., A.P. Kozikowski, G. Pinna, et al. 2008. The combination of huperzine A and imidazenil is an effective strategy to prevent diisopropyl fluorophosphate toxicity in mice. Proc Natl Acad Sci U S A. 105: 14169-14174.

19. Tuovinen, K. 2004. Organophosphate-induced convulsions and prevention of neuropathological damages. Toxicology. 196: 31-39.

20. Bajgar, J. 2004. Organophosphates/nerve agent poisoning: mechanism of action, diagnosis, prophylaxis, and treatment. Adv Clin Chem. 38: 151-216.

21. Mello, L.E., E.A. Cavalheiro, A.M. Tan, et al. 1993. Circuit mechanisms of seizures in the pilocarpine model of chronic epilepsy: cell loss and mossy fiber sprouting. Epilepsia. 34: 985-995.

22. Rice, A.C., C.L. Floyd, B.G. Lyeth, et al. 1998. Status epilepticus causes long-term NMDA receptor-dependent behavioral changes and cognitive deficits. Epilepsia. 39: 1148-1157. 23. Costa, M.S., J.B. Rocha, S.R. Perosa, et al. 2004. Pilocarpine-induced status epilepticus increases glutamate release in rat hippocampal synaptosomes. Neurosci Lett. 356: 41-44.

24. Tyler, A.L., J.M. Mahoney, G.R. Richard, et al. 2012. Functional network changes in hippocampal CA1 after status epilepticus predict spatial memory deficits in rats. J Neurosci. 32: 11365-11376.

25. Eddleston, M., N.A. Buckley, P. Eyer, et al. 2008. Management of acute organophosphorus pesticide poisoning. Lancet. 371: 597-607.

26. Chemical Hazards Emergency Medical Management. 2013. "Nerve Agents - Emergency Department/Hospital Management". http://chemm.nlm.nih.gov/na_hospital_mmg.htm\#top

27. de Araujo Furtado, M., F. Rossetti, S. Chanda, et al. 2012. Exposure to nerve agents: from status epilepticus to neuroinflammation, brain damage, neurogenesis and epilepsy. Neurotoxicology. 33: 1476-1490.

28. Helmstaedter, C. 2007. Cognitive outcome of status epilepticus in adults. Epilepsia. 48 Suppl 8: 85-90.

29. Neligan, A. \& S.D. Shorvon. 2011. Prognostic factors, morbidity and mortality in tonicclonic status epilepticus: a review. Epilepsy Res. 93: 1-10.

30. Ostrowsky, K. \& A. Arzimanoglou. 2010. Outcome and prognosis of status epilepticus in children. Seminars in pediatric neurology. 17: 195-200.

31. Read, M.I., A.A. Andreianova, J.C. Harrison, et al. 2014. Cardiac electrographic and morphological changes following status epilepticus: effect of clonidine. Seizure. 23: 55-61.

32. Rod, C.S. 2009. Status epilepticus in the developing brain: Long-term effects seen in humans. Epilepsia. 50: 32-33.

33. Phillips, K.F. \& L.S. Deshpande. 2016. Repeated low-dose organophosphate DFP exposure leads to the development of depression and cognitive impairment in a rat model of Gulf War Illness. Neurotoxicology. 52: 127-133.

34. Rosenstock, L., M. Keifer, W.E. Daniell, et al. 1991. Chronic central nervous system effects of acute organophosphate pesticide intoxication. The Lancet. 338: 223-227.

35. Savage, E.P., T.J. Keefe, L.M. Mounce, et al. 1988. Chronic neurological sequelae of acute organophosphate pesticide poisoning. Arch Environ Health. 43: 38-45. 
36. Levin, E.D., O.A. Timofeeva, L. Yang, et al. 2010. Early postnatal parathion exposure in rats causes sex-selective cognitive impairment and neurotransmitter defects which emerge in aging. Behav Brain Res. 208: 319-327.

37. Rauh, V.A., F.P. Perera, M.K. Horton, et al. 2012. Brain anomalies in children exposed prenatally to a common organophosphate pesticide. Proc Natl Acad Sci U S A. 109: 7871-7876.

38. Ruckart, P.Z., K. Kakolewski, F.J. Bove, et al. 2004. Long-term neurobehavioral health effects of methyl parathion exposure in children in Mississippi and Ohio. Environ Health Perspect. 112: 46-51.

39. Ivens, I.A., G. Schmuck \& L. Machemer. 1998. Learning and memory of rats after longterm administration of low doses of parathion. Toxicol Sci. 46: 101-111.

40. Dunn, C., S.B. Bird \& R. Gaspari. 2012. Intralipid fat emulsion decreases respiratory failure in a rat model of parathion exposure. Acad Emerg Med. 19: 504-509.

41. Gresham, C., C. Rosenbaum, R.J. Gaspari, et al. 2010. Kinetics and efficacy of an organophosphorus hydrolase in a rodent model of methyl-parathion poisoning. Acad Emerg Med. 17: 736-740.

42. Albuquerque, E.X., E.F. Pereira, Y. Aracava, et al. 2006. Effective countermeasure against poisoning by organophosphorus insecticides and nerve agents. Proc Natl Acad Sci U S A. 103: $13220-13225$.

43. Petrikovics, I., D. Papahadjopoulos, K. Hong, et al. 2004. Comparing therapeutic and prophylactic protection against the lethal effect of paraoxon. Toxicol Sci. 77: 258-262.

44. Todorovic, M.S., M.L. Cowan, C.A. Balint, et al. 2012. Characterization of status epilepticus induced by two organophosphates in rats. Epilepsy Res. 101: 268-276.

45. Ellison, C.A., Y. Tian, J.B. Knaak, et al. 2012. Human hepatic cytochrome P450-specific metabolism of the organophosphorus pesticides methyl parathion and diazinon. Drug Metab Dispos. 40: 1-5.

46. Eyer, F., V. Meischner, D. Kiderlen, et al. 2003. Human parathion poisoning. A toxicokinetic analysis. Toxicol Rev. 22: 143-163.

47. Jan, Y.H., J.R. Richardson, A.A. Baker, et al. 2015. Vitamin K3 (menadione) redox cycling inhibits cytochrome $\mathrm{P} 450$-mediated metabolism and inhibits parathion intoxication. Toxicol Appl Pharmacol. 288: 114-120.

48. Racine, R.J. 1972. Modification of seizure activity by electrical stimulation. II. Motor seizure. Electroencephalogr Clin Neurophysiol. 32: 281-294.

49. Steenland, K., B. Jenkins, R.G. Ames, et al. 1994. Chronic neurological sequelae to organophosphate pesticide poisoning. Am J Public Health. 84: 731-736.

50. Yamashita, M., M. Yamashita, J. Tanaka, et al. 1997. Human mortality in organophosphate poisonings. Veterinary and human toxicology. 39: 84-85.

51. Karki, P., J.A. Ansari, S. Bhandary, et al. 2004. Cardiac and electrocardiographical manifestations of acute organophosphate poisoning. Singapore Med J. 45: 385-389.

52. Munidasa, U.A., I.B. Gawarammana, S.A. Kularatne, et al. 2004. Survival pattern in patients with acute organophosphate poisoning receiving intensive care. Journal of toxicology. Clinical toxicology. 42: 343-347.

53. Burns, C.J., L.J. McIntosh, P.J. Mink, et al. 2013. Pesticide exposure and neurodevelopmental outcomes: review of the epidemiologic and animal studies. $J$ Toxicol Environ Health B Crit Rev. 16: 127-283. 
54. Raza, M., R.E. Blair, S. Sombati, et al. 2004. Evidence that injury-induced changes in hippocampal neuronal calcium dynamics during epileptogenesis cause acquired epilepsy. Proc Natl Acad Sci U S A. 101: 17522-17527.

55. DeLorenzo, R.J., D.A. Sun \& L.S. Deshpande. 2005. Cellular mechanisms underlying acquired epilepsy: the calcium hypothesis of the induction and maintainance of epilepsy.

Pharmacol Ther. 105: 229-266.

56. Nagarkatti, N., L.S. Deshpande \& R.J. DeLorenzo. 2009. Development of the calcium plateau following status epilepticus: role of calcium in epileptogenesis. Expert Rev Neurother. 9: 813-824.

57. Nagarkatti, N., L.S. Deshpande, D.S. Carter, et al. 2010. Dantrolene inhibits the calcium plateau and prevents the development of spontaneous recurrent epileptiform discharges following in vitro status epilepticus. Eur J Neurosci. 32: 80-88.

58. Filbert, M., E. Levine \& G. Ballough. 2005. Neuroprotection for nerve agent-induced brain damage by blocking delayed calcium overload: a review J Med CBR Def. 3.

59. Persiyantseva, N.A., K.R. Birikh, E.A. Dvoretskova, et al. 2008. Role of protein kinase C in $\mathrm{Ca}(2+)$ homeostasis disorders in cultured rat neurons during hyperstimulation of glutamate receptors. Bull Exp Biol Med. 145: 595-599.

60. Nagarkatti, N., L.S. Deshpande \& R.J. DeLorenzo. 2008. Levetiracetam inhibits both ryanodine and IP3 receptor activated calcium induced calcium release in hippocampal neurons in culture. Neurosci Lett. 436: 289-293.

61. Zolkowska, D., C.N. Banks, A. Dhir, et al. 2012. Characterization of seizures induced by acute and repeated exposure to tetramethylenedisulfotetramine. J Pharmacol Exp Ther. 341: 435-446.

62. Phillips, K.F., L.S. Deshpande, B. Huang, et al. 2015. Behavioral depression and memory impairment following organophosphate diisopropyl fluorophosphate induced status epilepticus in rats. Presented at American Epilepsy Society, Philadelphia, PA.

63. Battaglia, F.P., K. Benchenane, A. Sirota, et al. 2011. The hippocampus: hub of brain network communication for memory. Trends Cogn Sci. 15: 310-318.

64. Campbell, S. \& G. Macqueen. 2004. The role of the hippocampus in the pathophysiology of major depression. J Psychiatry Neurosci. 29: 417-426.

65. Deshpande, L.S., K. Phillips, B. Huang, et al. 2014. Chronic behavioral and cognitive deficits in a rat survival model of paraoxon toxicity. Neurotoxicology. 44: 352-357.

66. Brimfield, A.A. 2012. Chemicals of military deployments: revisiting Gulf War Syndrome in light of new information. Prog Mol Biol Transl Sci. 112: 209-230.

67. Odegard, T.N., C.M. Cooper, E.A. Farris, et al. 2013. Memory impairment exhibited by veterans with Gulf War Illness. Neurocase. 19: 316-327.

68. Deshpande, L.S., D.D. Limbrick, Jr., S. Sombati, et al. 2007. Activation of a novel injury-induced calcium-permeable channel that plays a key role in causing extended neuronal depolarization and initiating neuronal death in excitotoxic neuronal injury. $J$ Pharmacol Exp Ther. 322: 443-452.

69. Deshpande, L.S., D.A. Sun, S. Sombati, et al. 2008. Alterations In Neuronal Calcium Levels Are Associated With Cognitive Deficits After Traumatic Brain Injury. Neurosci Lett. 441: 115-119.

70. Bengtson, C.P. \& H. Bading. 2012. Nuclear calcium signaling. Adv Exp Med Biol. 970: 377-405. 
71. Baker, K.D., T.M. Edwards \& N.S. Rickard. 2013. The role of intracellular calcium stores in synaptic plasticity and memory consolidation. Neurosci Biobehav Rev. 37: 1211-1239.

72. Chadwick, W., N. Mitchell, B. Martin, et al. 2013. Therapeutic targeting of the endoplasmic reticulum in Alzheimer's disease. Curr Alzheimer Res. 9: 110-119.

73. Deshpande, L.S., N. Nagarkatti, J.M. Ziobro, et al. 2008. Carisbamate prevents the development and expression of spontaneous recurrent epileptiform discharges and is neuroprotective in cultured hippocampal neurons. Epilepsia. 49: 1795-1802.

74. Jett, D.A. 2010. Finding new cures for neurological disorders: a possible fringe benefit of biodefense research? Science translational medicine. 2: 23 ps12.

75. Jett, D.A. \& D.T. Yeung. 2010. The CounterACT Research Network: basic mechanisms and practical applications. Proceedings of the American Thoracic Society. 7: 254-256. 


\section{Figure Legends}

\section{Figure 1. Development of $\mathrm{Ca}^{2+}$ plateau and it's mechanism following OP induced SE}

A. Hippocampal CA1 $\left[\mathrm{Ca}^{2+}\right]$ i from age-matched control (white bar) and POX rats were isolated 1-h and 1, 7 and 30 days after SE (black bars). $\left[\mathrm{Ca}^{2+}\right]$ in POX-SE rats was significantly higher than control values at all the time-points and did not return to base-line even at 30-days post SE $\left(\mathrm{Ca}^{2+}\right.$ plateau). B. Hippocampal CA1 $\left[\mathrm{Ca}^{2+}\right]_{\mathrm{i}}$ from control rats (white bar), DFP rats (black bar), and DFP + MK-801 (grey bars) were isolated 1-h after SE. MK-801 pretreatment prevented the elevations in $\left[\mathrm{Ca}^{2+}\right]_{i}$ that occur following DFP induced SE. However, MK-801 treatment 1-h after DFP-induced SE did not significantly affect DFP-SE induced $\left[\mathrm{Ca}^{2+}\right]$ i elevations. C.

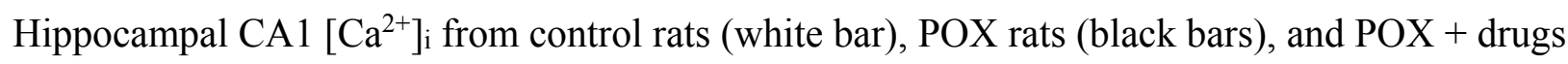
(grey bars) were isolated 48 -h after SE. $\left[\mathrm{Ca}^{2+}\right]_{\mathrm{i}}$ in neurons isolated from POX-SE rats treated with either dantrolene (DANT) or levetiracetam (LEV) or carisbamate (CRB) were significantly lower than POX SE rats (no drugs) values at the respective time point. All data represented as mean \pm SEM. ${ }^{*} p<0.05$ (Data in $1 A$ and $1 C$ reproduced from Deshpande, L.S., D.S. Carter, K.F. Phillips, et al. 2014. Development of status epilepticus, sustained calcium elevations and neuronal injury in a rat survival model of lethal paraoxon intoxication. Neurotoxicology. 44C: 17-26).

\section{Figure 2. Neuronal injury following POX induced SE}

Representative photomicrographs of Fluoro-Jade C (FJC) staining in the dentate gyrus-hilus region, parietal cortex, amygdala, and thalamus 2 days after POX SE. Scale bars, $200 \mu \mathrm{m}$. (Data previously published in : Deshpande, L.S., D.S. Carter, K.F. Phillips, et al. 2014. Development of status epilepticus, sustained calcium elevations and neuronal injury in a rat survival model of lethal paraoxon intoxication. Neurotoxicology. 44C: 17-26). 


\section{Figure 3. Chronic behavioral morbidities following POX induced SE}

Approximately 3-months following POX SE, surviving rats were tested on various behavioral assays for assessing symptoms of depression and memory impairments. A. Increased immobility time in POX SE rats during the Forced Swim Test indicative of behavioral despair. B. Decreased sucrose consumption in POX SE rats on the Sucrose Preference Test indicative of anhedonia (lack of feeling pleasure). C. Enhanced anxiety in POX SE rats as characterized by significantly less time spent in the open arm of the Elevated Plus Maze. D. Impaired recognition memory in POX SE rats on the Novel Object Recognition test as displayed significantly less time spent exploring the novel object. All data expressed as mean $\pm \mathrm{SEM},{ }^{*} p<0.05$, t-test, $\mathrm{n}=8$ rats. (Data adapted from: Deshpande, L.S., K. Phillips, B. Huang, et al. 2014. Chronic behavioral and cognitive deficits in a rat survival model of paraoxon toxicity. Neurotoxicology. 44: 352-357).

Figure 4. Development of the calcium plateau following OP induced SE and possible targets for countermeasures therapy

OP chemicals such as DFP or POX inhibit the enzyme acetylcholinesterase (AChE) initially producing a cholinergic crisis that propagates into self-sustaining SE and ultimately leads to glutamate excitotoxicity. Downstream activation of N-methyl-D-aspartate receptors (NMDA-R) leads to massive influx of $\mathrm{Ca}^{2+}$ ions into the post-synaptic neurons. Activation of $\mathrm{Ca}^{2+}$-induced $\mathrm{Ca}^{2+}$-release (CICR) mechanisms leads to release of $\mathrm{Ca}^{2+}$ into the cytoplasm from the endoplasmic reticulum (ER) via the ryanodine receptor (RyR) and the inositol-trisphosphate receptor $\left(\mathrm{IP}_{3} \mathrm{R}\right)$. While NMDA activation is required for genesis of $\mathrm{Ca}^{2+}$ plateau, the maintenance is dependent on sustained $\mathrm{Ca}^{2+}$ release via CICR mechanisms. After SE is terminated NMDA activation is shut off, but the $\mathrm{Ca}^{2+}$ release from ER continues due to a long lasting activation of CICR mechanisms. The $\mathrm{Ca}^{2+}$ plateau triggers neurodegenerative pathways leading to neuronal 
injury and activates nuclear signaling that can lead to neuronal plasticity that underlies chronic morbidities characterized by the development of acquired epilepsy, memory deficits, and psychiatric impairments. Inhibiting the critical targets $(1,2$ or 3$)$ in the $\mathrm{Ca}^{2+}$ plateau cascade with pharmacological agents (Dantrolene, Levetiracetam, or Ketamine) can exert neuroprotective effects and can decrease or prevent the development of the chronic neurological morbidities associated with OP SE survival. 


\section{Figure 1}

A

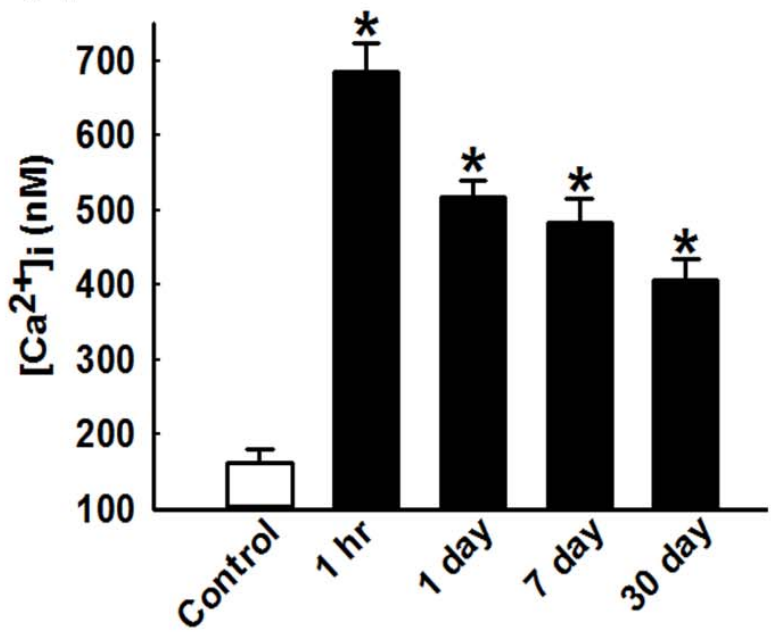

B

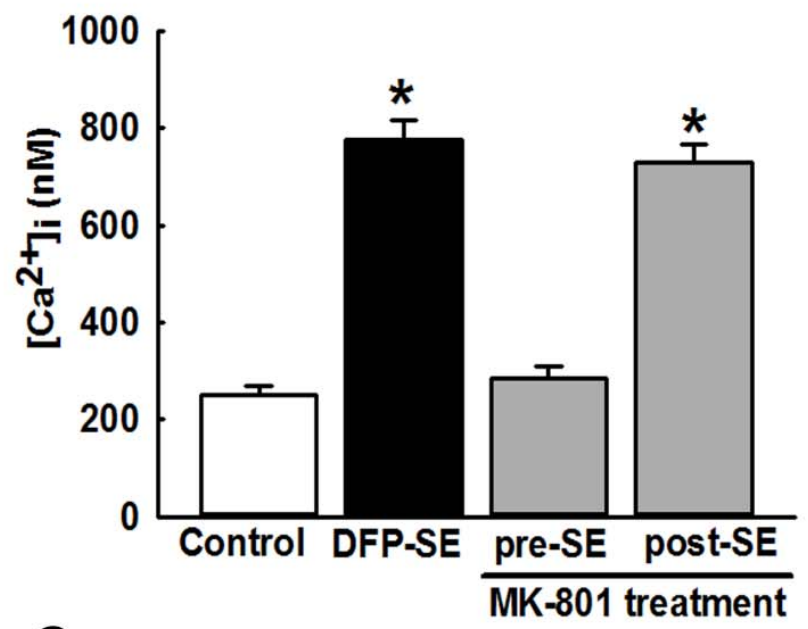

C

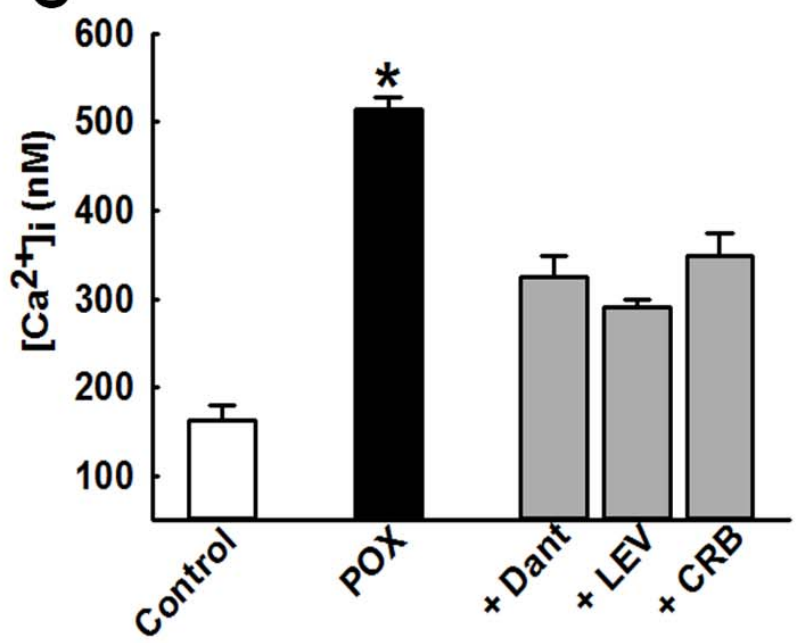


Figure 2

A

Dentate Gyrus Hilar Region

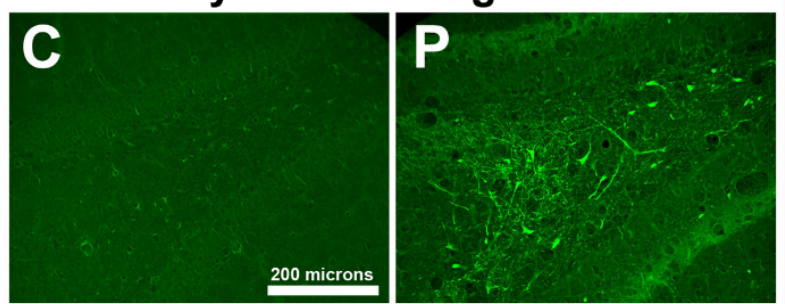

C

Parietal Cortex

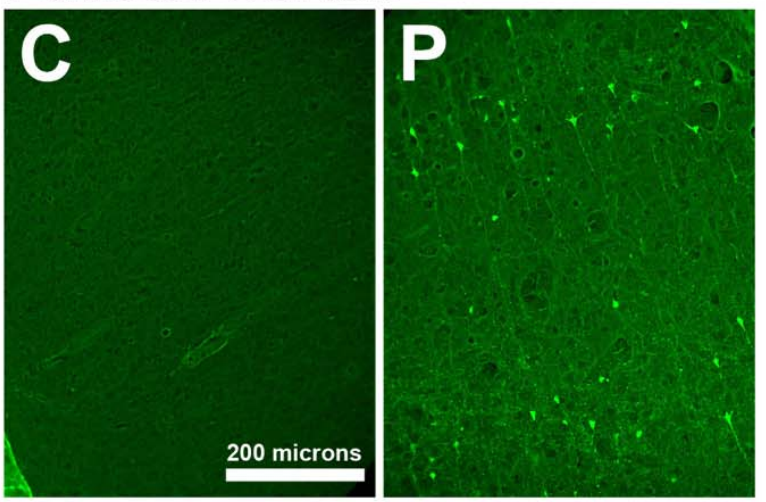

\section{B}

Thalamus

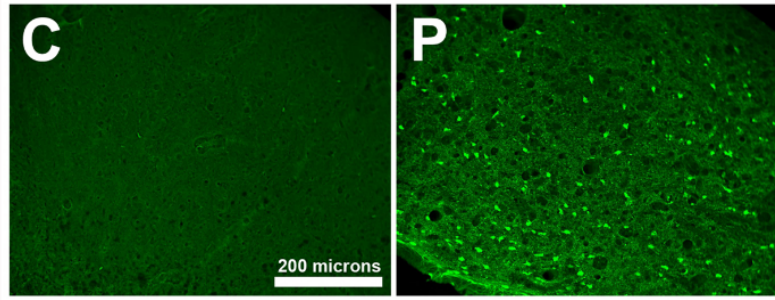

D

Temporal/Perirhinal Cortex

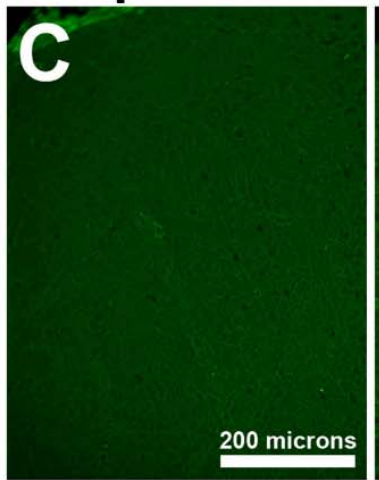

P

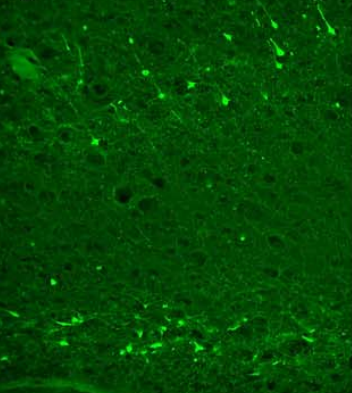


Figure 3

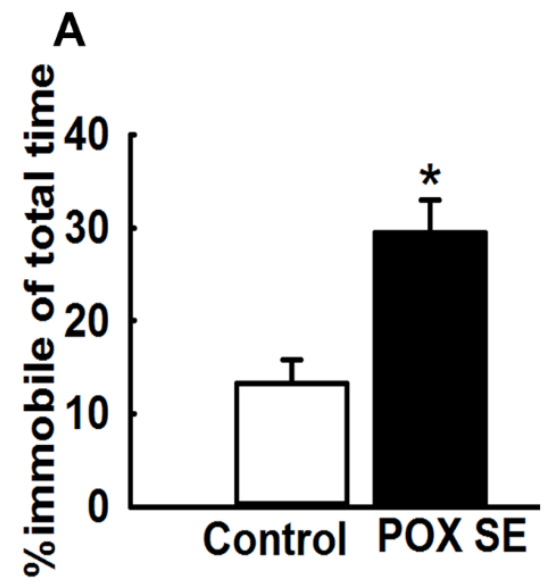

B

C
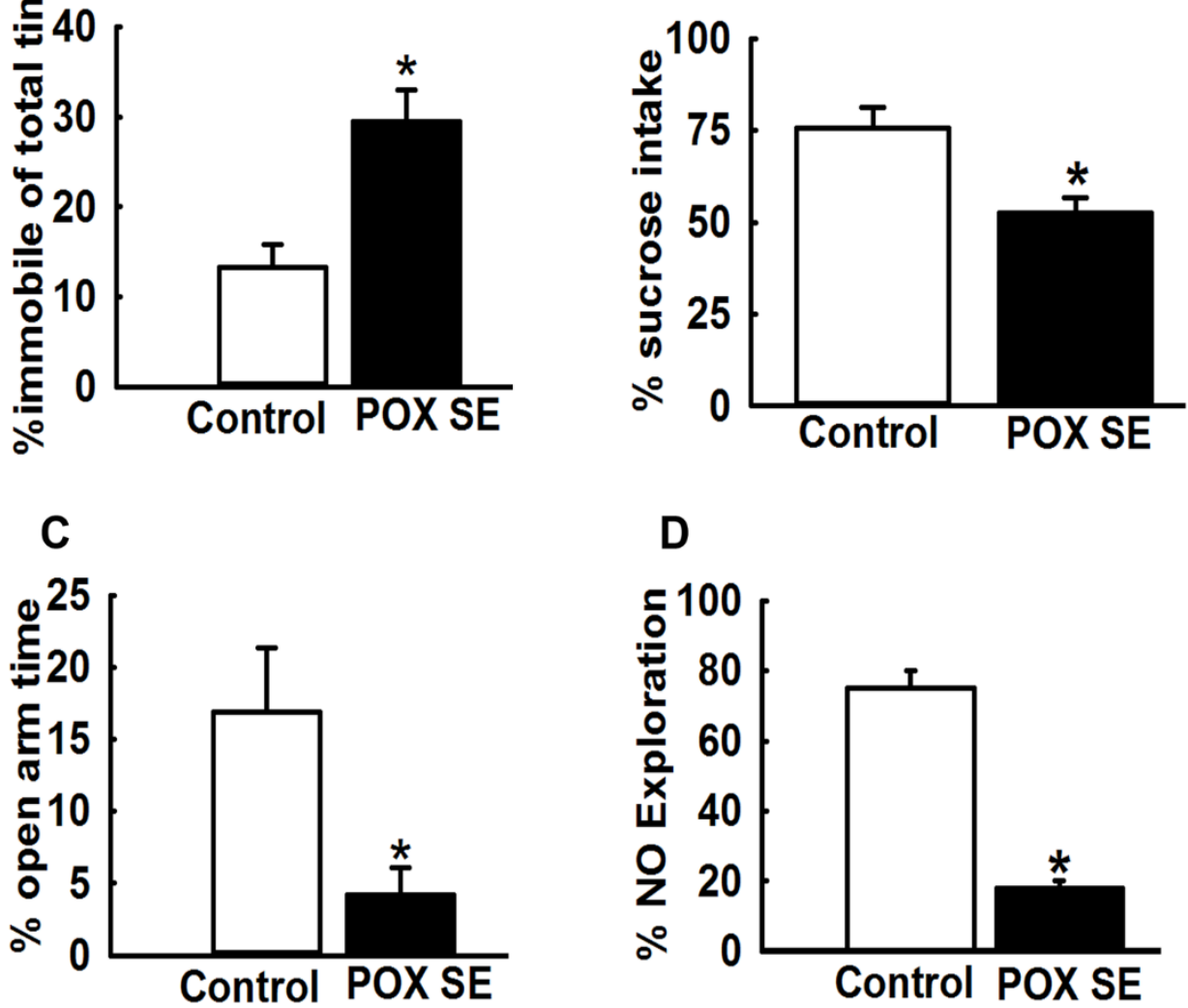


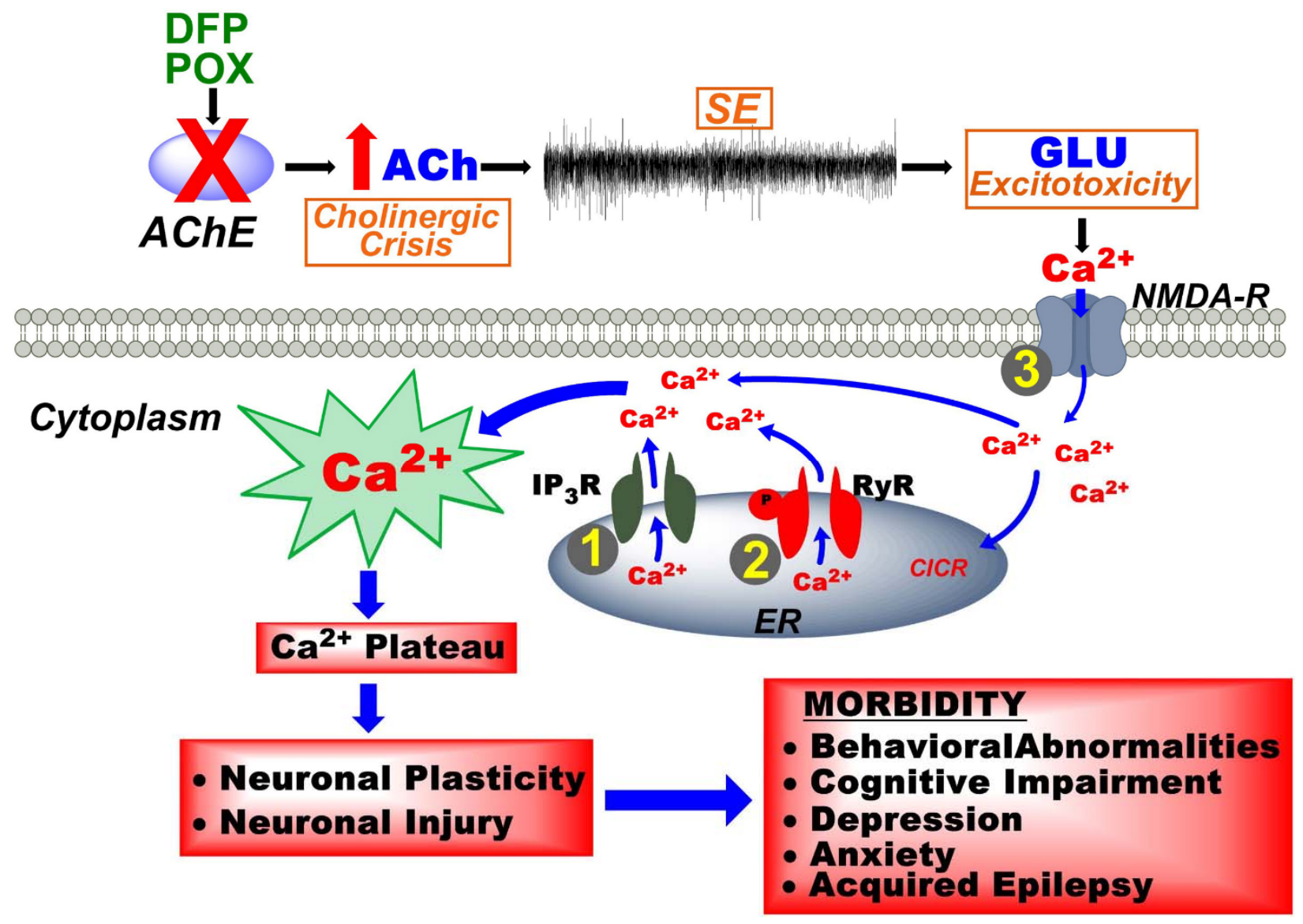

